\title{
紫外-可见分光光度法测定含铜废水中的铜离子
}

章子恒, 胡然, 马艺瑾, 孙兴蓉, 马思维, 朱忍, 曹秋娥, 周川华 ${ }^{*}$

云南大学化学科学与工程学院, 化学化工国家级实验教学示范中心(云南大学), 昆明 650091

摘要: 设计了一个通过紫外-可见分光光度法检测含铜废水中铜离子含量的本科教学实验。该实验基于芬顿反应原理, 检测液中铜离子的存在可催化过氧化氢分解产生羟基自由基, 后者将溶液中无色的 $3,3^{\prime}, 5,5^{\prime}$-四甲基联苯胺(TMB)氧 化为黄色的 $\mathrm{TMB}^{2+}$, 利用 $\mathrm{TMB}^{2+}$ 在 $452 \mathrm{~nm}$ 处的吸光度, 即可实现对含铜废水中铜离子的定量分析。本实验操作简便、 测定时间短、稳定性较好且安全环保, 符合本科教学实验的基本要求。

关键词: 芬顿反应; 铜离子检测; 紫外-可见分光光度法; 本科教学实验

中图分类号: G64; 0657.32

\section{An Experiment for Determining Copper(II) lons in Copper-Containing Sewage by UV-Vis Spectrophotometry}

\author{
Ziheng Zhang, Ran Hu, Yijin Ma, Xingrong Sun, Siwei Ma, Ren Zhu, Qiue Cao, Chuanhua Zhou * \\ National Demonstration Center for Experimental Chemistry and Chemical Engineering Education (Yunnan University), \\ School of Chemical Science and Technology, Yunnan University, Kunming 650091, China.
}

\begin{abstract}
A teaching experiment for determining copper(II) ions in copper-containing sewage by UV-Vis spectrophotometry is designed. The experiment is performed based on the Fenton reaction principle, where the presence of copper(II) ions in the assay solution catalyzes the decomposition of hydrogen peroxide to produce hydroxyl radicals. The latter oxidizes the colorless 3,3',5,5'-tetramethylbenzidine (TMB) in the solution to the yellow $\mathrm{TMB}^{2+}$, which can be used for quantitative analysis copper(II) ions in copper-containing sewage by measuring the absorbance of $\mathrm{TMB}^{2+}$ at $452 \mathrm{~nm}$. With easy operation, short measurement time, excellent stability, eco-friendliness, this experiment conforms to the basic requirements of undergraduate laboratory teaching.
\end{abstract}

Key Words: Fenton reaction; $\mathrm{Cu}(\mathrm{II})$ detection; Ultraviolet-visible spectrophotometry; Undergraduate teaching experiment

在本科基础化学教学实验中, 分析化学实验占有重要地位。其内容常包含分析化学基础实验与 兼具应用性和综合性的创新实验 ${ }^{[1]}$ 。目前, 传统分析化学实验教学模式存在诸如学生学习被动、兴

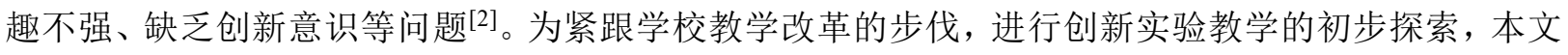
设计了一个仪器分析创新教学实验。该实验结合原有教学实验快速、准确的优点, 并将新方法引入 仪器分析实验教学中。这使其在呈现创新性的同时, 也在一定程度上将相对新颖的科研成果与本科 教学联系起来, 令学生在学习及操作的过程中感受到科研的鬼力, 从而激发学生的学习热情与兴趣, 进而充分提高学生的动手能力, 培养具有科学思维方法和创新能力的人才 ${ }^{[3]}$ 。 
本实验基于芬顿反应(Fenton reaction), 显色底物为 3,3',5,5'-四甲基联苯胺(TMB，3,3',5,5'Tetramethylbenzidine), 采用紫外-可见分光光度法测定废水中铜离子的含量。

芬顿反应为无机人名反应之一, 现今多用于污水处理、酶促反应、有机合成等诸多方面 ${ }^{[4]}$ 。铁、 铜、铝、铬等变价金属离子可作为芬顿反应的催化剂。其中，属铁基芬顿反应最为常见、应用最广; 而铜基芬顿反应的反应速率更快, 所适用的体系 $\mathrm{pH}$ 范围相对更宽 ${ }^{[5]}$ 。基于该反应, 可建立用于检测 芬顿反应体系中各物质的分析方法, 如: 比色法 ${ }^{[6]}$; 苂光光度法 ${ }^{[7]}$; 化学发光法 ${ }^{[8]}$; 分光光度法 ${ }^{[9]}$ 等。

$\mathrm{TMB}$ 常被用作酶促反应的显色底物 ${ }^{[10]}$, 在临床生化检验等方面应用广泛。该物质安全绿色, 适 合本科实验使用。

金属铜是冶金工业、电镀工业的重要原料，该类行业常排放大量含铜废水 ${ }^{[11]}$ 。废水中铜离子检

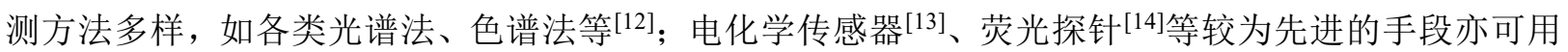
于检测铜离子。本实验采用紫外-可见分光光度法检测铜离子, 该方法是仪器分析教学的重要内容之 一，且相对简单、易理解，适于本科教学。

根据芬顿反应氧化TMB, 产生紫外-可见吸收的原理, 本团队设计优化此实验, 使之简单快捷, 便于操作。该实验灵敏度较高、重现性好, 满足本科教学实验基本要求。

本实验将催化反应引入本科教学实验, 且无需使用价格昂贵、容易变性失活的生物酶, 适用于 本科教学。芬顿反应在实验设计上灵活多变, 可以设计为检测不同物质(如过氧化氢、铜离子、铁离 子等其他变价金属离子)的分析检测方法。不仅利于加深学生对于紫外-可见分光光度法的理解; 还 可作为设计实验, 锻炼学生分析问题、解决问题的能力。本文将以铜离子检测为例, 建立一种基于 芬顿反应, 采用紫外-可见分光光度法检测铜离子的本科教学实验。

\section{1 实验部分}

\section{1 实验原理}

\subsection{1 铜基芬顿反应}

Zhi Shan等 ${ }^{[15]}$ 发现, 氯离子可显著加速铜基芬顿反应, 放大催化信号。在氯离子的加速作用下, 铜离子催化过氧化氢, 产生着基自由基。

$$
\mathrm{H}_{2} \mathrm{O}_{2} \stackrel{\mathrm{Cu}(\mathrm{II})}{\longrightarrow} \mathrm{HO} \cdot+\mathrm{OH}^{-}
$$

体系中羟基自由基将显色底物 $\mathrm{TMB}$ 氧化为蓝色的 $\mathrm{TMB}^{+}$, 后者在 $\mathrm{pH}<1$ 的条件下, 转变为稳定的 $\mathrm{TMB}^{2+}$, 如图1所示。 $\mathrm{TMB}^{2+}$ 呈黄色, 于 $452 \mathrm{~nm}$ 附近有最大吸收。

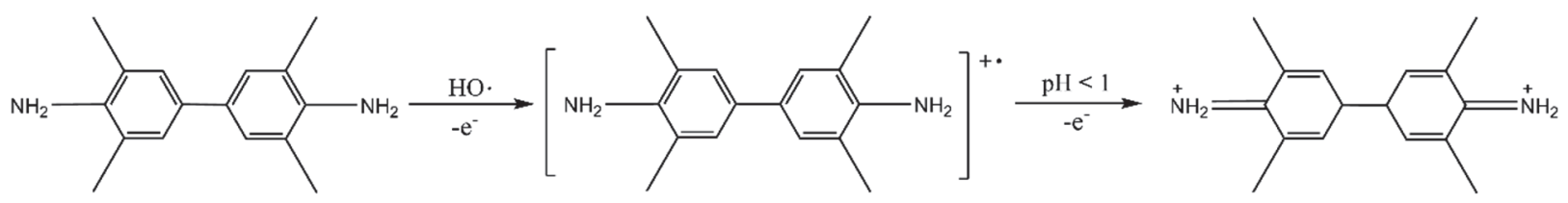

图1 TMB氧化机理示意图 ${ }^{[16]}$

\section{1 .2 紫外-可见光度分析及定量原理}

当平行单色光通过均匀、非散射的稀溶液时, 溶液的吸光度与其浓度间的关系满足Lambert-Beer 定律 ${ }^{[17]}$ 。其中, $k$ 为摩尔吸收系数, $b$ 为吸收池厚度。

$$
A=\kappa b c
$$

在相同波长下, 溶液中吸光物质一定时, 吸光度 $A$ 与溶液浓度 $c$ 成正比。

配制系列铜离子标准溶液, 将其与待测样品在相同底物条件下进行芬顿反应; 反应完成后, 测 定各体系紫外-可见吸收光谱, 记录最大吸收波长所对应的吸光度值; 绘制标准曲线, 将待测样品吸 光度值代入工作曲线方程中, 完成定量分析。 


\section{2 实验仪器与试剂}

\subsection{1 实验试剂}

超纯水, 无水乙醇(分析纯, 天津化学试剂厂), 硫酸铜储备液(以分析纯五水合硫酸铜配制, 天 津光复), 氯化钾(分析纯, 天津光复), 3,3',5,5'-四甲基联苯胺(分析纯, Adamas), 过氧化氢( $30 \%$, 西陇化工), 硫酸 $\left(98 \%\right.$, 西陇化工), $0.1 \mathrm{~mol} \cdot \mathrm{L}^{-1} \mathrm{pH}=4.5 \mathrm{HAc}-\mathrm{NaAc}$ 缓冲液(以分析纯冰乙酸及乙酸钠 配制, 天津化学试剂厂)。

\subsection{2 实验仪器}

UV-2600紫外-可见分光光度计(岛津, 日本), SYG-2数显恒温水浴锅(朗越, 中国), JP-010T超声 波清洗机(洁盟, 中国)。

\section{3 实验步骤}

\subsection{1 反应试剂配制}

1) 2.5、5.0、25.0 $\mu \mathrm{mol} \cdot \mathrm{L}^{-1} \mathrm{CuSO}_{4} \cdot 5 \mathrm{H}_{2} \mathrm{O}$ 储备液(由实验老师预先配制)。

2) $0.15 \mathrm{~mol} \cdot \mathrm{L}^{-1} \mathrm{KCl}(\mathrm{aq})$

准确称取 $1.1200 \mathrm{~g} \mathrm{KCl}$, 在干燥的烧杯中加水溶解, 转移至容量瓶, 并用超纯水定容至 $100.00 \mathrm{~mL}$, 得 $0.15 \mathrm{~mol} \cdot \mathrm{L}^{-1} \mathrm{KCl}(\mathrm{aq})$ 。

\section{3) $2.5 \mathrm{mmol} \cdot \mathrm{L}^{-1} \mathrm{TMB}$}

准确称取 $0.6000 \mathrm{~g} \mathrm{TMB}$ 于干燥烧杯中, 加入适量无水乙醇, 超声溶解, 转移至 $100 \mathrm{~mL}$ 容量瓶中, 以无水乙醇定容, 得 $25 \mathrm{mmol} \cdot \mathrm{L}^{-1} \mathrm{TMB}$ 。再移取 $10.00 \mathrm{~mL} 25 \mathrm{mmol} \cdot \mathrm{L}^{-1} \mathrm{TMB}$ 至 $100 \mathrm{~mL}$ 容量瓶, 以无水 乙醇定容, 最后得 $100.00 \mathrm{~mL} 2.5 \mathrm{mmol} \cdot \mathrm{L}^{-1} \mathrm{TMB}$ 溶液。

4) $1.0 \mathrm{~mol} \cdot \mathrm{L}^{-1} \mathrm{H}_{2} \mathrm{O}_{2}$

准确取 $10.00 \mathrm{~mL} 30 \% \mathrm{H}_{2} \mathrm{O}_{2}$ 于容量瓶中, 定容至 $100.00 \mathrm{~mL}$, 得 $1.0 \mathrm{~mol} \cdot \mathrm{L}^{-1} \mathrm{H}_{2} \mathrm{O}_{2}(\mathrm{aq})$ 。

5) $5.5 \mathrm{~mol} \cdot \mathrm{L}^{-1} \mathrm{H}_{2} \mathrm{SO}_{4}$

准确取 $30.00 \mathrm{~mL} 98 \% \mathrm{H}_{2} \mathrm{SO}_{4}$ 于已加入了适量水的烧杯中, 搅拌, 冷至室温, 转移至 $100 \mathrm{~mL}$ 容量 瓶, 定容, 最后得 $5.5 \mathrm{~mol} \cdot \mathrm{L}^{-1} \mathrm{H}_{2} \mathrm{SO}_{4}(\mathrm{aq})$ 。

6) 模拟含铜废水样品(由实验老师预先配制, 含铜 $6.0 \mu \mathrm{mol} \cdot \mathrm{L}^{-1}$ )。

7) $0.1 \mathrm{~mol} \cdot \mathrm{L}^{-1} \mathrm{pH}=4.5 \mathrm{HAc}-\mathrm{NaAc}$ 缓冲液(由实验老师预先配制)。

\subsection{2 过氧化氢浓度对反应的影响}

1) 取 10 个 $50 \mathrm{~mL}$ 比色管, 编号为空白、1-9。向各比色管中加入 $15.00 \mathrm{~mL} 5.0 \mu \mathrm{mol} \cdot \mathrm{L}^{-1} \mathrm{Cu}(\mathrm{II})$ 、 $5.00 \mathrm{~mL} 0.15 \mathrm{~mol} \cdot \mathrm{L}^{-1} \mathrm{KCl}(\mathrm{aq}) 、 5.00 \mathrm{~mL} 2.5 \mathrm{mmol} \cdot \mathrm{L}^{-1} \mathrm{TMB}$ 溶液, 充分振荡摇匀。

2) 向1-9号中加入 $0.25 、 0.50 、 0.75 、 1.00 、 2.00 、 3.00 、 4.00 、 5.00 、 6.00 \mathrm{~mL} 1.0 \mathrm{~mol} \cdot \mathrm{L}^{-1} \mathrm{H}_{2} \mathrm{O}_{2}(\mathrm{aq})$ ， 向空白组中加入 $5.00 \mathrm{~mL}$ 超纯水, 后快速以缓冲溶液定容至 $50.00 \mathrm{~mL}$ ，充分摇匀。

3) 将各比色管置于 $40{ }^{\circ} \mathrm{C}$ 下水浴加热 $30 \mathrm{~min}$, 每组独立计时。

4) 反应完成后立即取出各比色管, 加入 $5.00 \mathrm{~mL} 5.5 \mathrm{~mol} \cdot \mathrm{L}^{-1} \mathrm{H}_{2} \mathrm{SO}_{4}(\mathrm{aq})$, 终止反应, 摇匀。

5) 测定各组紫外-可见吸收光谱, 判断过氧化氢浓度对体系吸光度的影响情况。

\subsection{3 反应动态过程测定}

1) 取两个 $50 \mathrm{~mL}$ 比色管, 编号为 $1 、 2$ 。向各比色管中加入 $15.00 \mathrm{~mL} 25 \mu \mathrm{mol} \cdot \mathrm{L}^{-1} \mathrm{Cu}(\mathrm{II}) 、 5.00 \mathrm{~mL}$ $0.15 \mathrm{~mol} \cdot \mathrm{L}^{-1} \mathrm{KCl}(\mathrm{aq}) 、 5.00 \mathrm{~mL} 2.5 \mathrm{mmol} \cdot \mathrm{L}^{-1} \mathrm{TMB}$ 溶液, 充分振荡摇匀。

2) 向 1 号比色管中加入 $5.00 \mathrm{~mL} 1.0 \mathrm{~mol} \cdot \mathrm{L}^{-1} \mathrm{H}_{2} \mathrm{O}_{2}$ (aq), 以缓冲溶液定容至 $50.00 \mathrm{~mL}$, 充分摇匀, 并在室温下反应 $10 \mathrm{~min}$ 后, 测定紫外-可见吸收光谱, 确定 $\mathrm{TMB}^{+}$的最大吸收波长。

3) 向2号比色管中加入 $5.00 \mathrm{~mL} 1.0 \mathrm{~mol} \cdot \mathrm{L}^{-1} \mathrm{H}_{2} \mathrm{O}_{2}(\mathrm{aq})$, 以缓冲溶液定容至 $50.00 \mathrm{~mL}$, 充分摇匀后, 取 $1 \mathrm{~mL}$ 该混合溶液加入比色血中, 测定混合溶液中 $\mathrm{TMB}^{+}$最大吸收波长下的吸光度随时间变化曲线。

\subsection{4 测定实验步骤}

1) 取 9 个 $50 \mathrm{~mL}$ 比色管, 编号为空白、1-8, 作为反应容器。其中, 1-5 号为标准系列(标准 $1-5$ ); 
$6 、 7 、 8$ 号为三组平行样品(样品 $1-3)$ 。

2) 向空白组中加入 $5.00 \mathrm{~mL}$ 超纯水， 1-5号分别加入 $5.00 、 10.00 、 15.00 、 20.00 、 25.00 \mathrm{~mL}, 2.5$ $\mu \mathrm{mol} \cdot \mathrm{L}^{-1} \mathrm{Cu}(\mathrm{II})$ 。

3) 向6-8组中各加入 $5.00 \mathrm{~mL}$ 样品溶液。

4) 向各组中分别加入 $5.00 \mathrm{~mL} 0.15 \mathrm{~mol} \cdot \mathrm{L}^{-1} \mathrm{KCl}(\mathrm{aq})$ 。

5) 向各组中分别加入 $5.00 \mathrm{~mL} 2.5 \mathrm{mmol} \cdot \mathrm{L}^{-1} \mathrm{TMB}$ 溶液, 充分振荡摇匀。

6) 向各组中分别加入 $5.00 \mathrm{~mL} 1.0 \mathrm{~mol} \cdot \mathrm{L}^{-1} \mathrm{H}_{2} \mathrm{O}_{2}$ (aq), 后快速以缓冲溶液定容至 $50.00 \mathrm{~mL}$ 。

7) 上一步加入过氧化氢后, 立即开始计时(每组独立计时, 如表1所示), 并逐组放入恒温水浴锅 中, 在 $40{ }^{\circ} \mathrm{C}$ 下水浴加热 $30 \mathrm{~min}$ 。

8) 各组反应完成后立即取出, 迅速加入 $5.00 \mathrm{~mL} 5.5 \mathrm{~mol} \cdot \mathrm{L}^{-1} \mathrm{H}_{2} \mathrm{SO}_{4}(\mathrm{aq})$, 以终止反应, 充分摇 匀。

9) 测定各组紫外-可见吸收光谱, 绘制工作曲线, 计算样品中铜离子含量。

\subsection{5 计时方法实例}

表1 实验计时方法

\begin{tabular}{cccc}
\hline 编号 & 组别 & 开始时间 & 结束时间 \\
\hline 空白 & Blank & $00: 00$ & $30: 00$ \\
1 & Std.1 & $01: 00$ & $31: 00$ \\
2 & Std.2 & $02: 00$ & $32: 00$ \\
3 & Std.3 & $03: 00$ & $33: 00$ \\
4 & Std.4 & $04: 00$ & $34: 00$ \\
5 & Std.5 & $05: 00$ & $35: 00$ \\
6 & Samp.1 & $06: 00$ & $36: 00$ \\
7 & Samp.2 & $07: 00$ & $37: 00$ \\
8 & Samp.3 & $08: 00$ & $38: 00$ \\
\hline
\end{tabular}

\section{2 实验结果讨论}

\section{1 过氧化氢浓度的影响}

铜基芬顿反应主要与反应温度、反应时间、过氧化氢浓度、 $\mathrm{TMB}$ 浓度以及 $\mathrm{Cu}(\mathrm{II})$ 的浓度等因素有 关。本实验以过氧化氢为例, 对实验影响因素进行优化。固定其他反应条件, 测定不同浓度过氧化 氢对芬顿反应的影响。实验结果如图 $2(\mathrm{a})$ 所示, 过氧化氢浓度在 0.00 至 $0.02 \mathrm{~mol} \cdot \mathrm{L}^{-1}$ 范围内时, 吸光度 随浓度增加而增加; 当过氧化氢浓度超过 $0.02 \mathrm{~mol} \cdot \mathrm{L}^{-1}$ 后, 体系吸光度基本保持稳定。

\section{2 反应动力学曲线测定}

$\mathrm{TMB}^{+}$的最大吸收波长为 $654 \mathrm{~nm}$, 在此波长下测定反应动力学曲线。实验结果如图2(b)所示, 随 着铜基芬顿反应的进行, 体系中生成的 $\mathrm{TMB}^{+}$逐渐增加, 检测液 $654 \mathrm{~nm}$ 处的吸光度逐渐增大。随时间 的推移, 动力学曲线的斜率缓慢降低, 可能是由于随着催化反应的进行, 底物 $\mathrm{H}_{2} \mathrm{O}_{2}$ 的浓度降低, 产 物 $\mathrm{TMB}^{+}$浓度增加, 加速了逆反应的进行, 因此催化反应速率有所下降。

\section{3 吸收曲线绘制 $(\mathbf{4 0 0}-\mathbf{5 0 0} \mathrm{nm})$}

扫描1.3.4节中各组检测液的紫外-可见吸收光谱, 实验结果如图3所示, 检测液在 $452 \mathrm{~nm}$ 处有最 大吸收, 且随检测液中铜离子含量增加, 体系吸光度逐渐增大。 
(a)

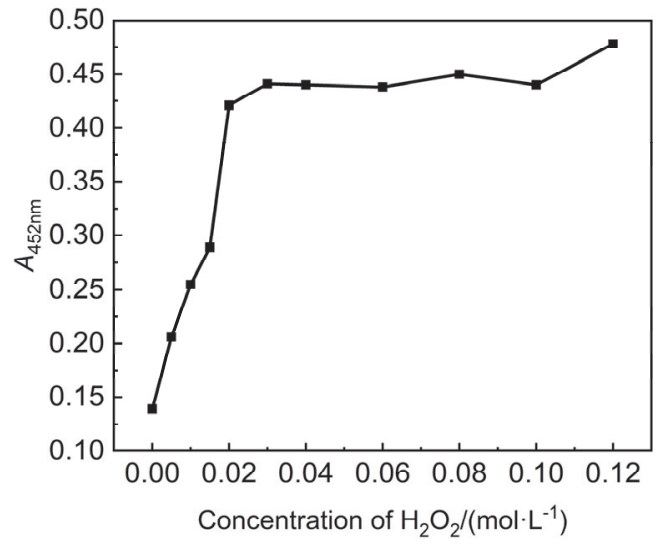

(b)

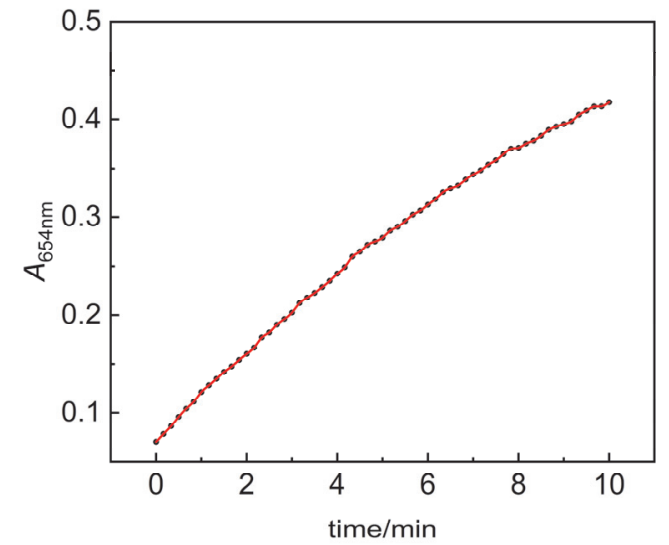

图 2 过氧化氢浓度对体系吸光度的影响(a)及芬顿反应动力学曲线(b)

(a)

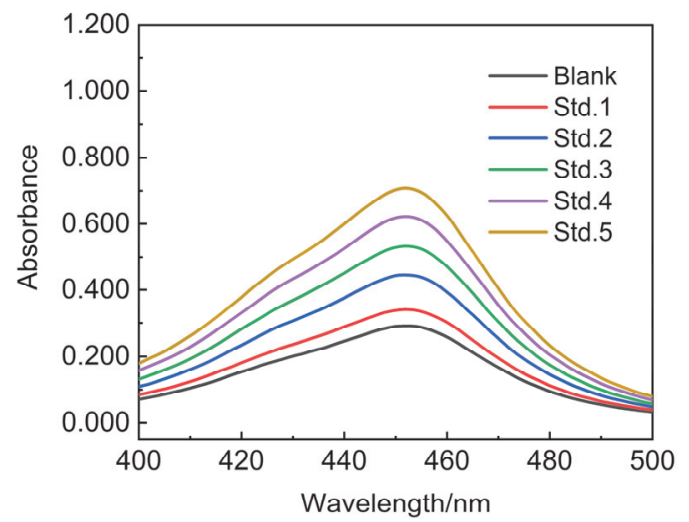

(b)

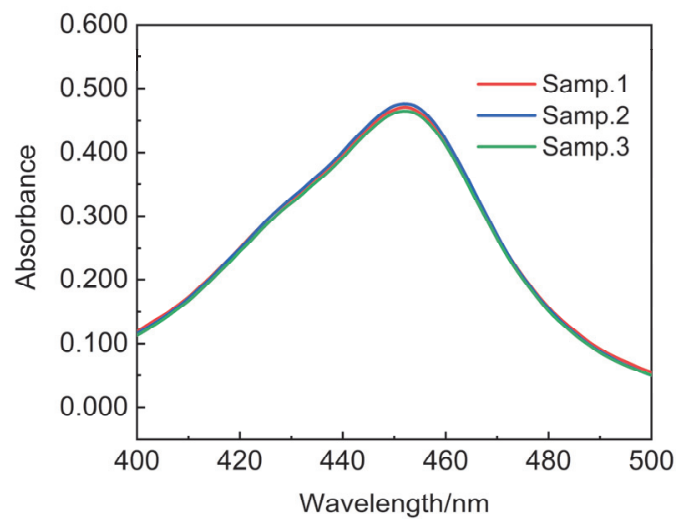

图3＼cjkstart采用芬顿反应体系检测不同铜离子标准溶液(a)及待测样品(b)的紫外-可见吸收光谱图 电子版为彩图

\section{4 工作曲线绘制}

由紫外-可见吸收光谱可知, 体系最大吸收波长为 $452 \mathrm{~nm}$ 。如表 2 所示, 记录体系中铜离子终浓 度 (以体系为 $50.00 \mathrm{~mL}$ 时计算) 以及对应的检测液在 $452 \mathrm{~nm}$ 处的吸光度值 $\left(A_{452 \mathrm{~nm}}\right)$ 。并以 $A_{452 \mathrm{~nm}}$ 作为纵 坐标, 铜离子浓度作为横坐标作图, 绘制工作曲线(图4)。

工作曲线方程为 $y=0.3636 x+0.2575$, 相关指数 $R^{2}=0.9987$, 线性关系良好, 实验稳定性较好。

表2 标准溶液中铜离子浓度-吸光度数据表

\begin{tabular}{ccc}
\hline Std. No. & $c_{\text {标 }}\left(\mu \mathrm{mol} \cdot \mathrm{L}^{-1}\right)$ & $A_{452 \mathrm{~nm}}$ \\
\hline 1 & 0.25 & 0.342 \\
2 & 0.50 & 0.445 \\
3 & 0.75 & 0.534 \\
4 & 1.00 & 0.622 \\
5 & 1.25 & 0.708 \\
\hline
\end{tabular}

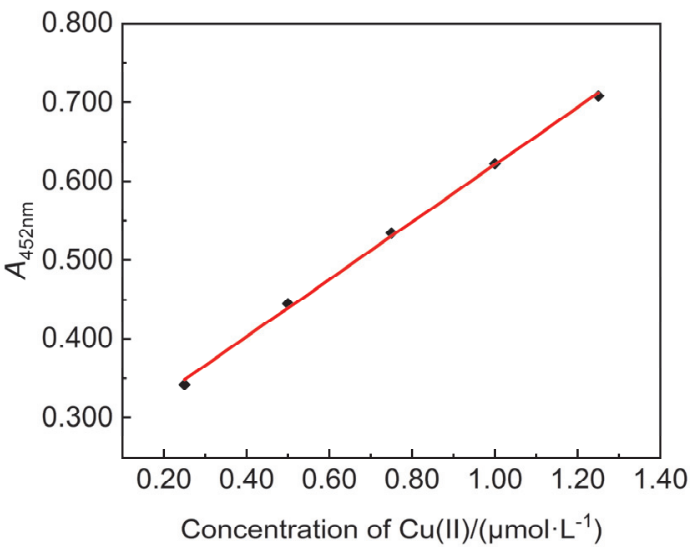

图4 铜离子检测工作曲线 


\section{5 样品浓度测定}

记录 $452 \mathrm{~nm}$ 下, 样品体系吸光度值(表3), 代入工作曲线方程, 得出体系为 $50.00 \mathrm{~mL}$ 时, 样品中 铜离子浓度, 并据此计算稀释前原始样品中铜离子浓度(表4)。

表3 平行样品浓度计算

\begin{tabular}{ccc}
\hline Samp. No. & $A_{452 \mathrm{~nm}}$ & $c_{\text {样 }}\left(\mu \mathrm{mol} \cdot \mathrm{L}^{-1}\right)$ \\
\hline 1 & 0.471 & 0.587 \\
2 & 0.476 & 0.601 \\
3 & 0.465 & 0.571 \\
\hline
\end{tabular}

表4 样品原始浓度计算及误差分析

\begin{tabular}{cccc}
\hline $\bar{c} /\left(\mu \mathrm{mol} \cdot \mathrm{L}^{-1}\right)$ & $\mathrm{RSD}$ & 原浓度 $/\left(\mu \mathrm{mol} \cdot \mathrm{L}^{-1}\right)$ & 相对误差 \\
\hline 0.586 & $1.50 \%$ & 5.86 & $2.33 \%$ \\
\hline
\end{tabular}

结果表明, 模拟废水样品中铜离子平均原始浓度为 $5.86 \mu \mathrm{mol} \cdot \mathrm{L}^{-1}$, 与预设值相近, 相对误差为 $2.33 \%$, 测定结果较准确。三次检测的相对标准偏差为 $1.50 \%$, 满足仪器分析实验基本要求。

\section{6 讨论}

本实验误差主要来自以下方面: 一是溶液配制误差, 二是溶液移取误差, 三是反应时长误差, 四是仪器系统误差。实际操作本实验时, 实验者需准确配制溶液及移取试剂, 还需尽可能按照实验 方法准确计时, 以确保各组反应时长一致。

\section{7 注意事项}

1) 反应需尽量准确计时, 以尽可能保证各组反应时长一致, 减小误差;

2) 需注意TMB、 $\mathrm{H}_{2} \mathrm{O}_{2}$ 的低温避光保存, 最好现配现用;

3) 因盐酸溶液中, 氯离子含量较高, 本实验不宜换用盐酸来终止反应。

\section{3 实验条件讨论}

\section{1 氯离子加速铜基芬顿反应}

铜基芬顿反应体系中，氯离子的存在可以显著加速铜基芬顿反应 ${ }^{[15]}$ 。我们比较了不同浓度氯离 子对铜基芬顿反应的放大效果, 实验结果如图 $5(\mathrm{a})$ 所示, 在 $1.5 \mu \mathrm{mol} \cdot \mathrm{L}^{-1}$ 铜离子的存在下, 随着氯离 子浓度的增加, 检测液 $452 \mathrm{~nm}$ 处的吸光度逐渐增大(红色柱状图); 当体系中不含铜离子时, $\mathrm{TMB}^{2+}$ $452 \mathrm{~nm}$ 处的吸光度较小(黑色柱状图), 证明氯离子的存在可以加速铜基芬顿反应。

\section{2 方法的选择性}

为了考查方法的选择性，采用该方法对相同浓度的其他几种常见的金属离子进行检测，所得到 的实验结果如图 $5(\mathrm{~b})$ 所示, 这些干扰物所得到的检测信号较小, 只有 $\mathrm{Cu}(\mathrm{II})$ 的存在才能够使检测液在 $452 \mathrm{~nm}$ 处产生较大的信号。虽然 $\mathrm{Fe}(\mathrm{II})$ 等变价金属也能够催化芬顿反应, 但是由于铜基芬顿反应的反 应速率更快, 且氯离子的存在进一步加速了铜基芬顿反应, 所以该方法具有较好的选择性。

(a)

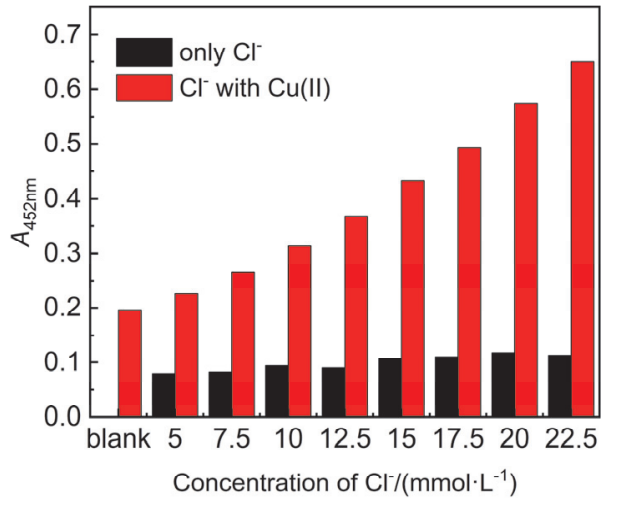

(b)

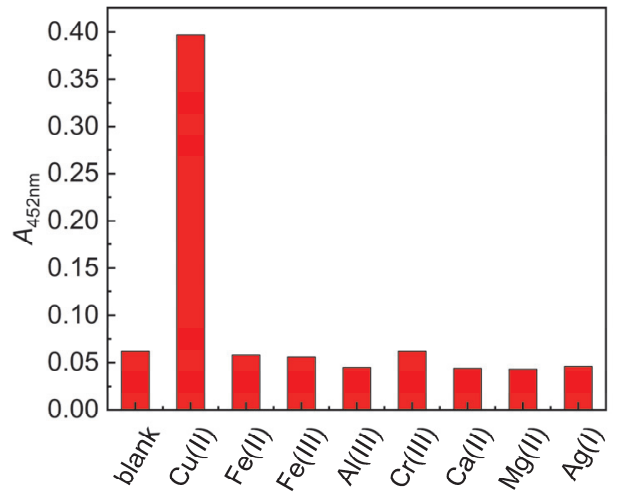

图5 不同浓度氯离子对反应的影响(a)及几种常见金属离子对反应的影响(b) 电子版为彩图 


\section{4 特点及特色声明}

向本科生介绍了该种快速、简单、可操作性强的铜离子检测方法, 使其认识、理解并实际操作 芬顿反应, 同时帮助学生学习掌握紫外-可见分光光度法。

1) 本实验安全, 可重复性强, 操作方便, 适用于本科教学;

2) 实验稳定性好, 重现性较强;

3) 反应试剂毒性较小, 相对安全;

4) 经改进后, 可设计成用于 $\mathrm{Fe}^{2+} 、 \mathrm{Cl}^{-} 、 \mathrm{H}_{2} \mathrm{O}_{2}$ 等物质测定的检测实验。

\section{5 结语}

本实验简单快捷, 重现性较好, 对铜离子的检测相对准确。向本科生介绍芬顿反应-TMB显色体 系, 并实际应用其完成定量分析, 拓宽实验者知识面。此外, 本实验采用紫外-可见分光光度法进行 测定, 有利于帮助学生巩固课程知识, 掌握仪器结构与操作 ${ }^{[18]}$, 一定程度上有助于教学改革的落 实 ${ }^{[19]}$ 。本实验还可以拓展设计成用于检测芬顿反应体系中其他物质(如: 过氧化氢、铁离子等)的本 科教学实验。

\section{参 考 文 献}

[1] 杨素芳. 科技视界, 2019, No. 22, 127.

[2] 柳玉英, 王平, 王粤博, 刘青, 萄红桃, 张天, 范慧清. 实验室研究与探索, 2018, No. 4, 217.

[3] 范乃英, 侯海鸽, 尹贻东, 曹尔新, 牛晓宇. 实验室研究与探索, 2010, 29 (7), 258.

[4] 张德莉, 黄应平, 罗光富, 刘德富, 马万红, 赵进才. 环境化学, 2006, No. 2, 121.

[5] 方梦媛. 铜基类芬顿催化剂的制备及其光助氧化性能研究(硕士学位论文). 上海: 上海交通大学, 2019.

[6] 徐向荣, 王文华, 李华斌. 生物化学与生物物理进展, 1999, No. 1, 3.

[7] 徐向荣, 王文华, 李华斌. 分析化学, 1998, No. 12, 1460.

[8] 韩鹤友, 何治柯, 曾云鹗. 分析化学, 1999, No. 8, 890 .

[9] 刘立明, 刘丽虹, 宋功武, 方光荣. 湖北大学学报(自然科学版), 2002, No. 4, 326.

[10] Rye, D. B.; Saper, C. B.; Wainer, B. H. J. Histochem. Cytochem. 1984, 32 (11), 1145.

[11] 李博, 刘述平. 矿产综合利用, 2008, No. 5, 33 .

[12] 付海䂀, 刘威, 张春辉, 李艳. 理化检验(化学分册), 2012, 48 (4), 496.

[13] 陈宏硕, 张雪莉, 崔传金. 食品研究与开发, 2016, 37 (18), 205.

[14] 胡明明, 焚江莉, 李宏林, 帕哈丁, 泽耀东, 彭孝军. 分析化学, 2011, 39 (8), 1195

[15] Zhi, S.; Mingsheng, L.; Li, W. Chem. Commun. 2016, 52, 2087.

[16] Josephy, P. D.; Eling, T.; Mason, R. P. J. Biol. Chem. 1982, 257 (7), 3669.

[17] 武汉大学. 分析化学(上册). 第6版. 北京: 高等教育出版社, 2016.

[18] 陈滕, 徐锋, 杨菁, 麻秀萍. 广东化工, 2019, 46 (20), 148.

[19] 靳明, 景雪拳, 韦丽红, 白国义, 杨瑜涛. 实验室研究与探索, 2020, 39 (9), 204. 